tatsächliche Inhalt erfüllt. Dies gilt besonders für die letzten Kapitel, die Informationen über bekannte außersprachliche Umstände wiederholen, ohne neue Überlegungen zu der Beziehung zwischen der Sprache und der außersprachlichen Geschichte zu bieten. Die lange schriftliche Geschichte des Ungarischen hätte mit ihren Dokumenten die Voraussetzungen dafür liefern können. Vielleicht will man den Leser selbst zu diesen Überlegungen anregen, da die entsprechenden
Themen einem Handbuch der Sprachgeschichte beigefügt wurden. Als Lehrbuch ist das Werk eine hervorragende Einführung in das Thema, auch wenn leider viele Grundlagen vor allem aus der Geschichte der Morphologie fehlen. Das gleiche gilt natürlich auch für das Werk als „Handbuch”: Nicht alles ist darin zu finden, und viele Dinge werden in den verschiedenen Teilen des Buchs aus unterschiedlichen Perspektiven behandelt.

Ulla-Maija Forsberg

\title{
Von Sprachkontakten zum Sprachtod oder zur Superdiversität?
}

<https://doi.org/ | 0.33339/fuf.79538>

Linguistic Genocide or Superdiversity? New and Old Language Diversities. Ed. by Reetta Toivanen and Janne Saarikivi. Linguistic Diversity and Language Rights: 14. Multilingual Matters. Bristol - Buffalo • Toronto 2016.362 S.

In der Uralistik ist man ständig damit konfrontiert, dass Sprachen, die wir untersuchen, bedroht sind und aussterben. Das Problem beschränkt sich nicht allein auf die uralischen Sprachen, denn unterschiedlichen Schätzungen zufolge sind 50-90 \% der Sprachen der
Welt vom Aussterben bedroht. Diese Entwicklung wurde vor allem seit dem 20. Jahrhundert durch die Kolonisation, die Modernisierung und die Globalisierung beschleunigt. Als Folge dieser Faktoren sind auch neue Kreolsprachen, Mischsprachen sowie neue ethnische Identitäten, Städter- und Einwanderergruppen entstanden. Die Welt hat sich verändert, und die Veränderung wird immer schneller. Dieser Prozess bringt nicht nur den Tod von Sprachen mit sich, sondern auch eine neuartige - und weitgehend unerforschte - ethni- 
sche und sprachliche Variation, die sich nicht nur auf die gesprochene Sprache beschränkt, sondern auch die geschriebene Sprache berührt.

Licht auf diese Fragen wirft das von Reetta Toivanen und Janne Saarikivi herausgegebene Buch, das schon im Titel zwei Perspektiven einander gegenüberstellt: Sprachtod oder Superdiversität. Das Vorwort der beiden umreißt den Inhalt des Werks unter der Überschrift Neue und alte Sprachdiversitäten: Variation und Bedrohung der Sprache in sich wandelnden Minoritätsgemeinschaften. Neben der herrschenden Landessprache werden einige dominierende Weltsprachen auf Kosten der lokalen Sprache und der Mehrsprachigkeit favorisiert. Die stärkste dieser auch als Killer bezeichneten Sprachen ist natürlich das Englische. Es ist in gewisser Weise widersprüchlich, dass die moderne Ausbildung im Zuge ihrer Verbreitung die Vielfalt der Meinungen und Weltanschauungen reduziert und alles vereinheitlicht, was in ihren Einflussbereich gerät. (Dasselbe ist auch in der Kultur zu beobachten: Wenn man der Volksmusik der Mansen oder Mari ein universales Disko-Comping hinzufügt - was heute so oft geschieht - werden sie einander gleicht und verlieren Besonderheit.)

Die 12 Beiträge des Werks sind in drei thematische Gruppen gegliedert, und von einigen Ausnahmen abgesehen steht in ihrem Mittelpunkt eine uralische Sprache. Der erste Teil trägt den Titel Language communities or networks of communication? Old and new linguistic diversity. Im ersten Kapitel dieses Teils befassen sich Niko Partanen und Janne Saarikivi mit der Fragmentierung des Karelischen und der karelischen Gemeinschaft sowie mit den damit verbundenen sprachlichen Prozessen wie der intensiven Entlehnung, dem Kodewechsel, dem Kodekopieren und der Entstehung neuer Konstruktionen, die allesamt die sprachliche Variation vergrößern. Während die Variation in der früheren „ursprünglichen“ Sprechergemeinschaft vornehmlich areal, sozial und situativ war, besteht sie in der heutigen erlöschenden Gemeinschaft aus Kodewechsel und Mischsprache. Die Statistiken über die Sprachauffassungen und den Sprachgebrauch der Karelier, die Partanen und Saarikivi bei ihren Feldstudien in Russisch-Karelien gesammelt haben, enthüllen die Unausweichlichkeit des Verschwindens der Sprache. Wie sie unter Hinweis auf Dell Hymes feststellen, bedeutet kommunikative Kompetenz nicht nur die Beherrschung der Grammatik und des Wortschatzes einer Sprache, sondern setzt auch die Teilnahme am Leben 
der Sprechergemeinschaft voraus, denn nur durch Teilnahme erlernt ein Kind die sozialen Dimensionen der Sprache.

Heini Lehtonen befasst sich mit der Gestaltung der Sprache und der Wechselwirkung in multiethnischer Umgebung bei Jugendlichen in Helsinki, die sie bereits in ihrer Dissertation untersucht hat. Ihr Material stammt aus zwei Sekundarstufen, deren Schüler rund zwanzig verschiedene Erstsprachen sprechen. Lehtonen untersucht in ihrem Artikel What's up Helsinki? Linguistic diversity among suburban adolescents vor allem, wie und in welchen Funktionen die Jugendlichen Wörter und Ausdrücke anderer Sprachen in unterschiedlichen sozialen Situationen verwenden und wie sie sich mit deren Hilfe in der Gemeinschaft positionieren. Ihrer Ansicht nach sollte man dem sog. Superdiversitätsparadigma gemäß Diversität als Ausgangspunkt der sprachlichen Analyse und nicht als Ausnahme betrachten. Der in den Artikel eingefügte knappe Vergleich mit dem Helsinkier Slang des 20. Jahrhunderts bleibt recht oberflächlich.

Boglárka Janurik untersucht in ihrem Artikel den ersanisch-russischen Kodewechsel in den Sendungen der mordwinischen Radioanstalt Vajgel' und stützt sich dabei auf das sog. Kontinuitätsmodell.
Diesem Modell zufolge kann man einen Text recht mechanisch in fünf Stadien gliedern, davon ausgehend, welches die Matrixsprache und welches die eingebettete Sprache ist und in welchem Verhältnis beide vorkommen. Janurik schreibt ersanisch mit lateinischen Buchstaben nach den Transliterationsregeln des Russischen, also gewissermaßen so, als handle es sich um Russisch; dadurch wird u.a. die Palatalität der Konsonanten nur am Wortende angezeigt, und diese Schreibweise ist meines Erachtens nicht akzeptabel. Hätte man nicht eher kyrillische Buchstaben verwenden sollen?! - Christian Pischlöger kartiert in seinem Artikel die Verwendung des Udmurtischen in den sozialen Medien und vergleicht sie mit den kymrischen Internetdomänen. Er macht besonders auf den Purismus aufmerksam, der in den udmurtischen Medien begegnet.

Der zweite Teil des Bandes befasst sich mit der Standardisierung von Sprachen und Ethnizitäten und fragt, ob dies eine "mission impossible" sei. Den Anfang macht Hanna Lantto, die die gegenwärtige und historische Wechselwirkung zwischen den beiden Sprachen von Bilbao, dem Baskischen und dem Spanischen, betrachtet. Der Einfluss begann bereits vor zweitausend Jahren mit dem Kontakt zwischen Lateinisch und Baskisch und setzt 
sich bis heute fort, nachdem sich das örtliche Latein im Lauf der Zeit zum Spanischen entwickelt hatte. Das Ergebnis ist häufiger Kodewechsel und gegenseitiger Einfluss der Sprachen aufeinander. Lantto zufolge ist schon in spanischsprachigen Dokumenten aus dem 13. Jahrhundert eine starke baskische Interferenz zu beobachten - und schon damals herrschte eine puristische Einstellung zu diesem Phänomen.

Oksana Myshlovska analysiert in ihrem Artikel Nationalising fluid and ambiguous identities den Status und die Ethnizität der ukrainischen Minderheit in Russland und der russischen Minderheit in der Westukraine und untersucht, welche Rolle Status und Ethnizität im politischen und soziolinguistischen Diskurs haben. Sprache ist nicht dasselbe wie Nationalität, sondern beide und die Auffassungen von ihnen variieren in vielerlei Art.

Erika Katjaana Sarivaara untersucht den Wandel der saamischen Sprache und der Identität der Saamen: Von der Assimilation zu Beginn des 20. Jahrhunderts ist man auf den Weg zur Revitalisierung gelangt. Anhand der Äußerungen der Befragten umreißt sie die in Finnland geführte Diskussion über die Frage, wer "richtige“ Saamen sind (oder sein dürfen). - Reetta Toivanen analysiert die Situation im mehrsprachigen und von mehreren Kulturen geprägten Inari. Ihrer Ansicht nach basieren persönliche oder Gruppenidentitäten nicht mehr auf Geschlecht, Muttersprache, Ethnizität oder Herkunft, sondern werden durch einen internationalen Bezugsrahmen geformt. Die Identität ist mit anderen Worten heute ein globales Projekt, das den örtlichen Ansprüchen angepasst wird. Toivanen beschreibt den Effekt des internationalen Bezugsrahmens der Menschenrechte auf die Beziehungen zwischen den verschiedenen Bevölkerungsgruppen in Inari, Nord-, Skolt- und Inarisaamen sowie Finnen, und die durch die Bewegung der indigenen Völker beflügelte Entwicklung der Identitäten der Saamen. Finnland hat das Übereinkommen 169 der ILO über indigene Völker bekanntlich immer noch nicht ratifiziert.

Der dritte Teil des Bandes ist der Wiederbelebung der Sprache gewidmet: Language revitalisation: Protection standards or tolerance for variation. Konstantin Zamyatin untersucht den Unterricht der Minderheitensprachen in Russland im Licht der Europäischen Charta der Regional- oder Minderheitensprachen. Russland hat die Charta 2001 unterzeichnet, aber nicht ratifiziert. Die Schulreformen der letzten zwei Jahrzehnte haben die Stellung der Minderheitensprachen an den 
Schulen verschlechtert. Der Unterricht in der Minderheitensprache ist freiwillig und wird nur angeboten, wenn die Kinder und ihre Eltern ihn fordern. In einer Situation, in der die Minderheitensprache geringschätzig angesehen wird und ihr Erlernen keinen sichtbaren Nutzen verspricht, fordern die meisten diesen Unterricht nicht, zumal die Auffassung vorherrscht, Zweisprachigkeit sei schädlich. Nur in den Republiken, in denen mehr als die Hälfte der Einwohner zu einer Minderheitsbevölkerung gehört, nämlich in Tschuwaschien, Tatarstan und Baschkortostan, wird die Minderheitensprache teilweise auch als Unterrichtssprache verwendet, in allen anderen Gebieten kann sie nur Unterrichtsfach sein. Interessanterweise ist in diesen Republiken auch die Position der dort gesprochenen kleineren Sprachen besser als in den nach ihnen benannten Republiken. Mit anderen Worten werden zum Beispiel Mari und Udmurtisch in den marischen und udmurtischen Dorfschulen in Tatarstan und Baschkortostan als Unterrichtssprache verwendet, nicht aber in den Schulen der Republiken Mari und Udmurtien.

Johanna Laakso untersucht den Begriff Sprachdiversität in ihrem Artikel Metadiversity, or the uniqueness of the lambs. Zwar wird in vielen europäischen Staaten die
Sprachdiversität als Ziel der Sprachpolitik genannt, doch wird sie eher geduldet als gefördert, es handelt sich also nur um leere Worte. Laakso stützt sich bei ihren Thesen weitgehend auf die Resultate des von ihr geleiteten ELDIA-Projekts (European Language Diversity for All). Dieselbe umfangreiche Erhebung von ELDIA verwenden Ulriikka Puura und Outi Tánczos in ihrem Artikel über den Revitalisierungsdiskurs des Karelischen und Wepsischen. 2004 wurde ein Gesetz über staatliche Unterstützung für das Karelische, Wepsische und Finnische erlassen, und das Wepsische erhielt im Jahr 2000 den Status der zahlenmäßig kleinen indigenen Völker Russlands. Aktive Maßnahmen zur Wiederbelebung der Sprachen gibt es jedoch kaum, und statt von Revitalisierung spricht man von Bewahrung. Die Bewahrung der Sprache wird mit der traditionellen Kultur verknüpft und tritt nicht als selbständige Frage hervor. Die Identität der Menschen ist eher lokal als national.

Das Thema der Untersuchung von Svetlana Edygarova sind die permischen Sprachen und die Ideologie, die deren Standardsprache hervorhebt: Nach dem Muster des Russischen wird im öffentlichen Gebrauch keine dialektale Ausdrucksweise, sondern nur die sog. Standardsprache akzeptiert. Wie 
Edygarova feststellt, ist die Standardsprache für viele eine fremde Sprachform, denn sie wird selten verwendet, in der Schule unzureichend gelehrt, und die Sprecher haben keine emotionale Bindung an sie. Aus demselben Grund werden auch viele in den letzten Jahrzehnten geschaffene Neologismen abgelehnt. Im Hinblick auf die Erhaltung der Sprache vertritt diese Einstellung eine falsche Strategie: Wenn man den Sprechern eine fehlerhafte oder dialektale Sprache attestiert, werden sie nicht ermutigt, die Sprache zu verwenden, sondern ihr eher entfremdet.

Das Werk Linguistic genocide or superdiversity untersucht den Wandel der Minderheitensprachen und ihrer Sprachgemeinschaften. Wie die Herausgeber Saarikivi und Toivanen in ihrem Vorwort feststellen, streben sie keine Dekonstruktion der traditionellen Sprachgemeinschaften an, sondern wollen zeigen, dass der Begriff Superdiversität jene neuen sprachlichen und ethnischen Identitäten widerspiegelt, die entstehen, wenn die alte Lebensweise der Sprachgemeinschaften schwindet. Insgesamt bietet das Werk eine neuartige Perspektive auf die Situation der uralischen Sprachen und deren Erforschung und öffnet ein neues Fenster für die Untersuchung des Wandels dieser Sprachen.

Sirkka Saarinen

\section{Etymologische Erörterung ungarischer Wörter}

LÁszló HonTI: A magyar és a nyugati ótörök szókészleti kapcsolatairól [Über die lexikalischen Beziehungen zwischen dem Ungarischen und dem Westalttürkischen]. Segédkönyvek a nyelvészet tanulmányozásához 196. Budapest: Tinta könyvkiadó 2017. 218 S.
László Honti, der Grand Old Man der Ostjakologie und der finnischugrischen Sprachgeschichte, ließ sich durch das 2011 von András Róna-Tas und Árpád Berta veröffentlichte zweibändige Wörterbuch (West Old Turkic. Turkic loanwords in Hungarian) dazu anregen, eine Stellungnahme zu den in diesem 